\title{
Experimental and Theoretical Studies of Sodium Cation Interactions with the Acidic Amino Acids and Their Amide Derivatives
}

A. L. Heaton, R. M. Moision, and P. B. Armentrout*

\section{Supplementary Information}

One table of geometries and relative energies for zwitterionic conformations of $\mathrm{Na}^{+}$(Asp), $\mathrm{Na}^{+}$(Asn), $\mathrm{Na}^{+}$(Glu), and $\mathrm{Na}^{+}(\mathrm{Gln})$. Four figures showing low-energy conformations of Asn, Gln, $\mathrm{Na}^{+}\left(\right.$Asn), $\mathrm{Na}^{+}(\mathrm{Gln})$, and zwitterionic conformations of $\mathrm{Na}^{+}$(Asp), $\mathrm{Na}^{+}$(Asn), $\mathrm{Na}^{+}(\mathrm{Glu})$, and $\mathrm{Na}^{+}(\mathrm{Gln})$.

FIGURE S1. Ground and excited state conformations of neutral Asn and Gln calculated at the B3LYP/6-311+G(d,p) level of theory. Hydrogen bond lengths are shown in $\AA$.

FIGURE S2. Ground and excited state conformations of sodiated Asn and Gln calculated at the B3LYP/6-311+G(d,p) level of theory. Hydrogen bond lengths are shown in $\AA$.

FIGURE S3. Lowest energy zwitterionic conformations of sodiated Asp and Asn calculated at the B3LYP/6-311+G(d,p) level of theory. Hydrogen bond lengths are shown in $\AA$.

FIGURE S4. Lowest energy zwitterionic conformations of sodiated Glu and Gln calculated at the B3LYP/6-311+G(d,p) level of theory. Hydrogen bond lengths are shown in $\AA$. 
TABLE S1: Bond Distances $(\AA)$, Bond Angles $\left({ }^{\circ}\right)$, and Relative Energies (kJ/mol) for Low-energy Zwitterionic Structures of Sodiated Asx and Glx

\begin{tabular}{|c|c|c|c|c|c|c|c|c|}
\hline Species & & $\mathrm{r}\left(\mathrm{Na}^{+}-\mathrm{O}\right)^{\mathrm{b}}$ & $\mathrm{r}\left(\mathrm{Na}^{+}-\mathrm{O}\right)^{\mathrm{c}}$ & $\angle\left(\mathrm{O}^{\mathrm{b}} \mathrm{NaO}^{\mathrm{b}}\right)$ & $\angle\left(\mathrm{O}^{\mathrm{b}} \mathrm{NaO}^{\mathrm{c}}\right)$ & B3LYP & B3Р86 & MP2(full) \\
\hline $\mathrm{Na}^{+}$(Asp) & {$\left[\mathrm{CO}_{2}^{-}\right] \mathrm{gtt}$} & $2.294,2.314$ & & 58.2 & & 24.5 & 19.2 & 24.7 \\
\hline $\mathrm{Na}^{+}$(Asn) & {$\left[\mathrm{CO}_{2}^{-}\right] \mathrm{gt}$} & $2.295,2.299$ & & 58.4 & & 21.6 & 15.8 & 23.5 \\
\hline \multirow[t]{4}{*}{$\mathrm{Na}^{+}(\mathrm{Glu})$} & {$\left[\mathrm{CO}_{2}^{-}\right] \mathrm{ggtt}$} & 2.287, 2.307 & & 58.3 & & 7.5 & 0.8 & 9.9 \\
\hline & {$\left[\mathrm{CO}_{2}{ }^{-}\right] \operatorname{tgtt}$} & $2.278,2.315$ & & 58.4 & & 8.0 & 1.8 & 13.0 \\
\hline & {$\left[\mathrm{CO}_{2}^{-}\right] \mathrm{tctt}$} & 2.282, 2.312 & & 58.4 & & 29.4 & 24.2 & 29.9 \\
\hline & {$\left[\mathrm{CO}_{2}^{-}, \mathrm{CO}\right]$ gggt } & $2.350,2.360$ & 2.309 & 57.0 & $80.3,90.0$ & 32.5 & 26.5 & 25.5 \\
\hline \multirow[t]{4}{*}{$\mathrm{Na}^{+}(\mathrm{Gln})$} & {$\left[\mathrm{CO}_{2}^{-}\right] \mathrm{ggt}$} & $2.285,2.295$ & & 58.5 & & 7.5 & -0.2 & 9.0 \\
\hline & {$\left[\mathrm{CO}_{2}^{-}\right] \operatorname{tgt}$} & $2.274,2.305$ & & 58.6 & & 7.6 & 0.6 & 11.9 \\
\hline & {$\left[\mathrm{CO}_{2}^{-}\right] \mathrm{tct}$} & $2.279,2.302$ & & 58.6 & & 29.0 & 23.0 & 28.5 \\
\hline & {$\left[\mathrm{CO}_{2}{ }^{-}, \mathrm{CO}\right] \mathrm{gtt}$} & $2.356,2.359$ & 2.267 & 57.0 & $80.5,91.8$ & 33.8 & 29.1 & 29.6 \\
\hline
\end{tabular}

${ }^{a}$ Structures are named by their binding sites to the sodium cation (in brackets) and by the series of dihedral angles starting from the carboxylic acid carbon of the backbone and going to the terminal R-group hydrogen for the acids and terminal R-group nitrogen for the amides. The dihedral angles are distinguished as cis (c, for angles between $\left.0^{\circ}-45^{\circ}\right)$, gauche $\left(\mathrm{g}, 45^{\circ}-135^{\circ}\right)$, or trans $\left(\mathrm{t}, 135^{\circ}-\right.$ $180^{\circ}$ ). Structures calculated at B3LYP/6-311+G(d,p) level of theory. Single point energies determined at B3LYP, B3P86, MP2(full)/6-311+G(2d,2p) //B3LYP/6-311+G(d,p). ${ }^{\text {b }}$ Equivalent oxygens of the $\mathrm{CO}_{2}{ }^{-}$group in the amino acid backbone. ${ }^{\mathrm{c}}$ Carbonyl oxygen of the amino acid side chain. 
FIGURE S1.

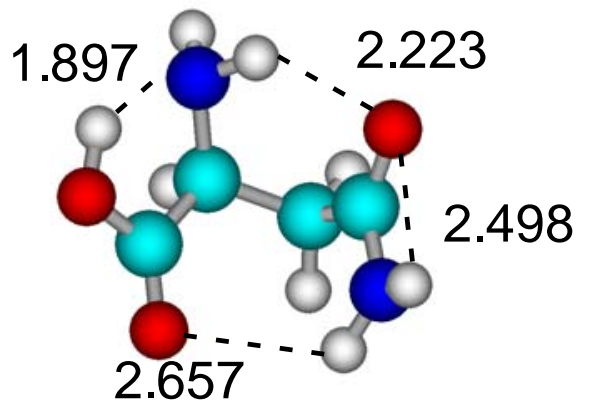

Asn-ctgg

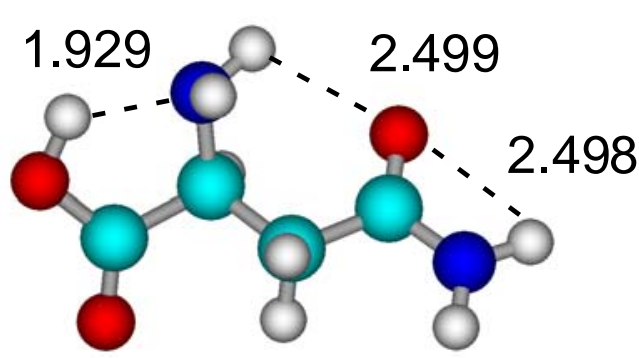

Asn-cttt
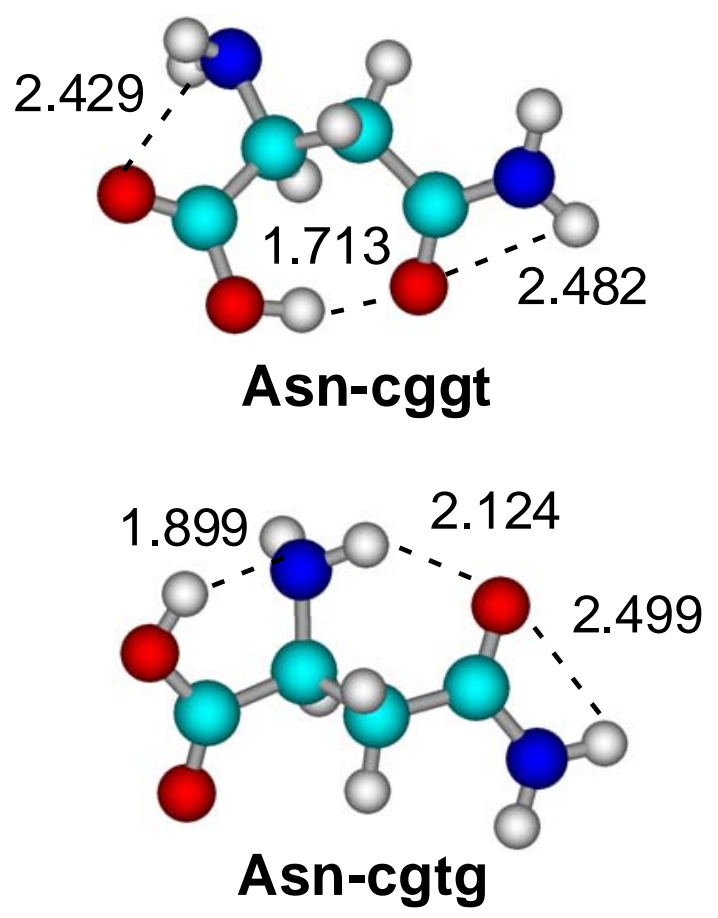

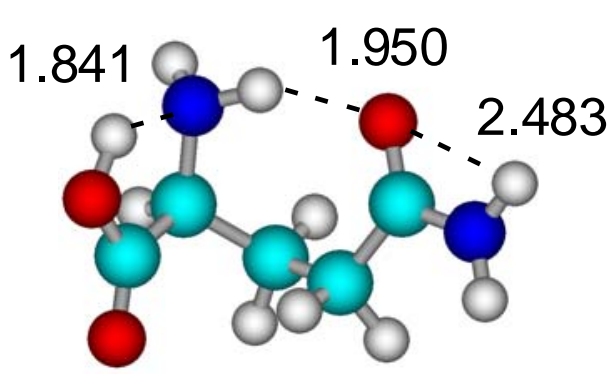

Gln-ctggt

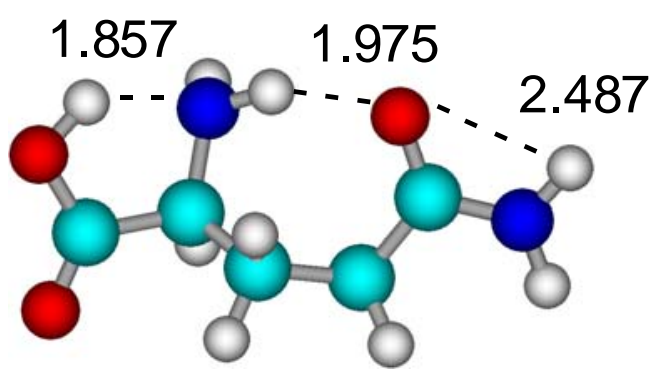

Gln-cgtgt

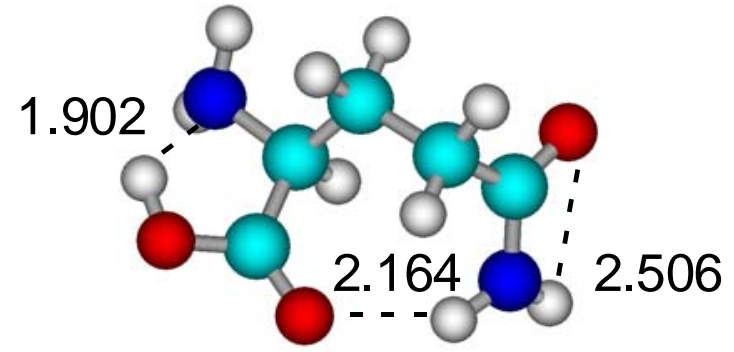

Gln-cg,ggg

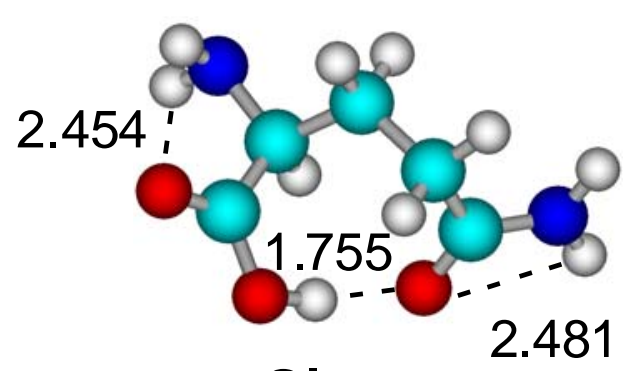

Gln-cg.ggg 
FIGURE S2.

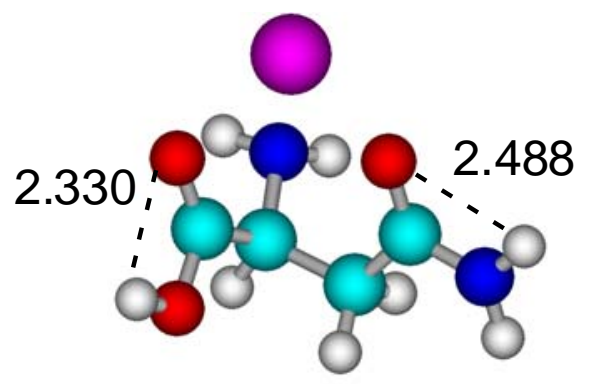

$\mathrm{Na}^{+}($Asn) $[\mathrm{N}, \mathrm{CO}, \mathrm{CO}] \operatorname{tggt}$

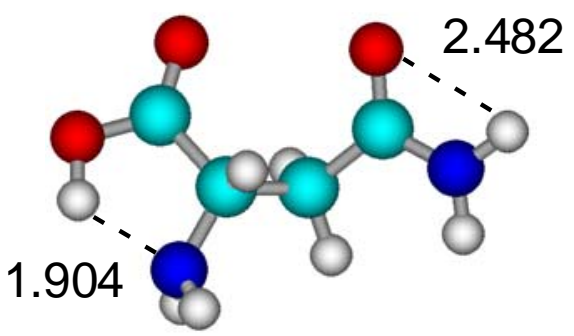

$\mathrm{Na}^{+}$(Asn) $[\mathrm{CO}, \mathrm{CO}] \mathrm{ctg}_{+} \mathrm{g}$

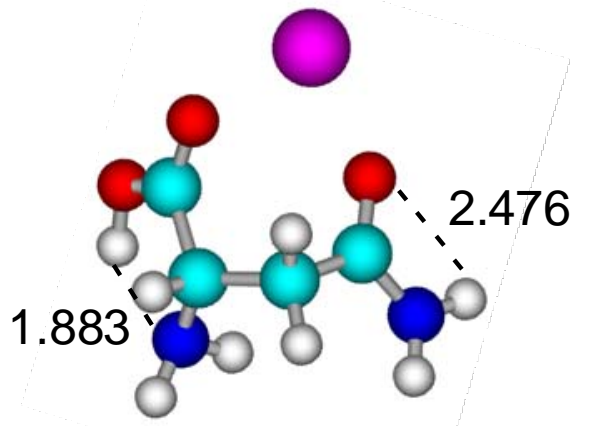

$\mathrm{Na}^{+}$(Asn) $[\mathrm{CO}, \mathrm{CO}] \operatorname{ctg} . \mathrm{g}$

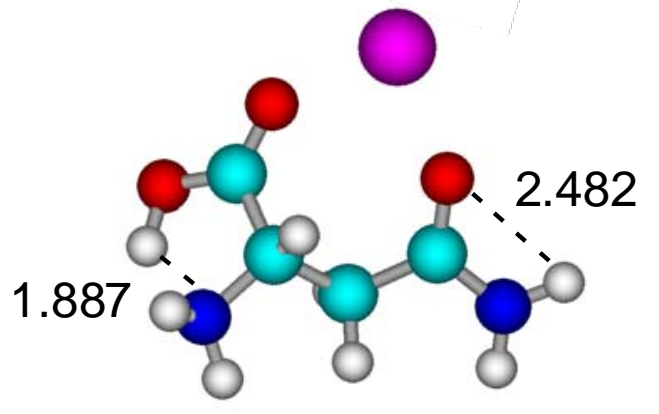

$\mathrm{Na}^{+}$(Asn) $[\mathrm{CO}, \mathrm{CO}] \mathrm{cggt}$

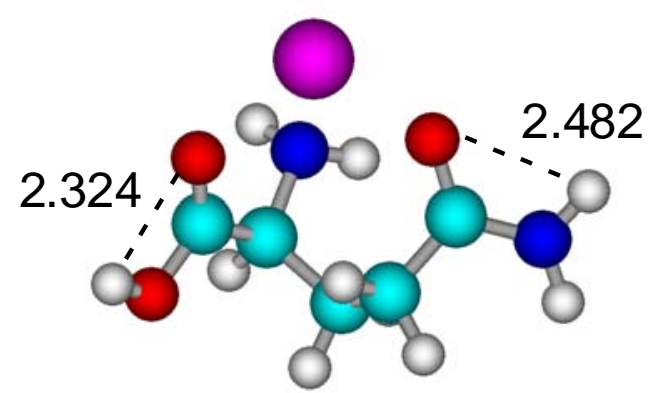

$\mathrm{Na}^{+}(\mathrm{GIn})[\mathrm{N}, \mathrm{CO}, \mathrm{CO}] \operatorname{tg} g \mathrm{~g} . \mathrm{g}$

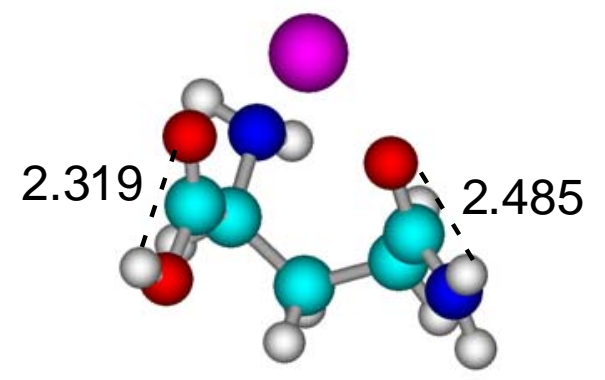

$\mathrm{Na}^{+}(\mathrm{Gln})[\mathrm{N}, \mathrm{CO}, \mathrm{CO}] \operatorname{tggg}_{+} \mathrm{g}$

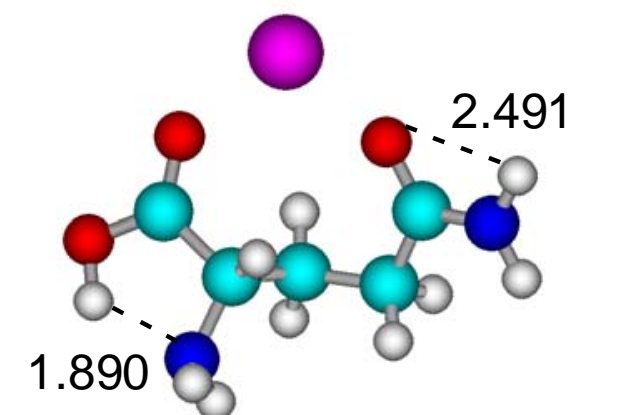

$\mathrm{Na}^{+}(\mathrm{GIn})[\mathrm{CO}, \mathrm{CO}] \mathrm{cgtgt}$

1.894

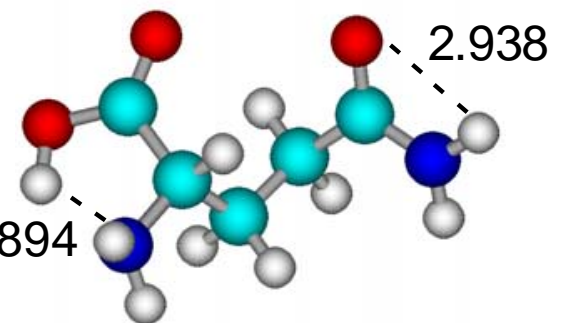

$\mathrm{Na}^{+}(\mathrm{G}$ In) $[\mathrm{CO}, \mathrm{CO}] \mathrm{cgggg}$ 
FIGURE S3.
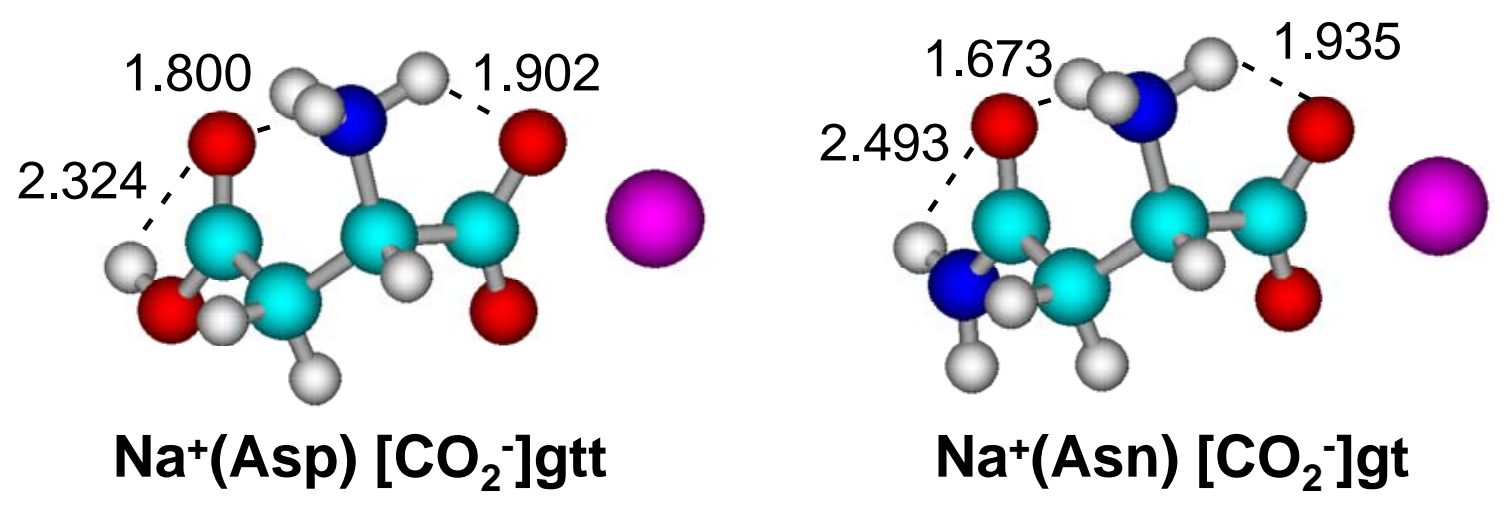
FIGURE S4.
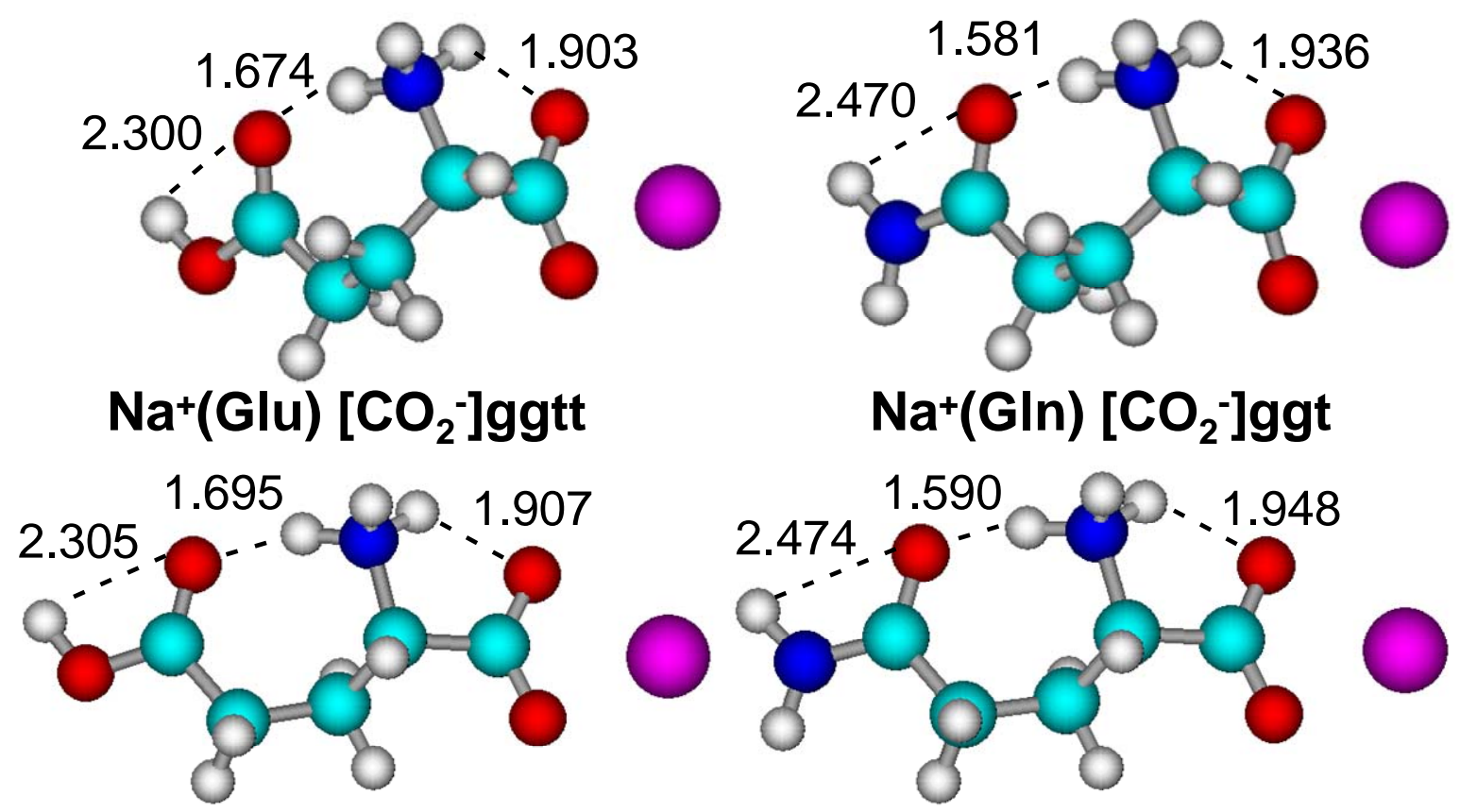

\section{$\mathrm{Na}^{+}(\mathrm{Glu})\left[\mathrm{CO}_{2}^{-}\right] \mathrm{tgtt}$}

$\mathrm{Na}^{+}(\mathrm{Gln})\left[\mathrm{CO}_{2}^{-}\right] \mathrm{tgt}$
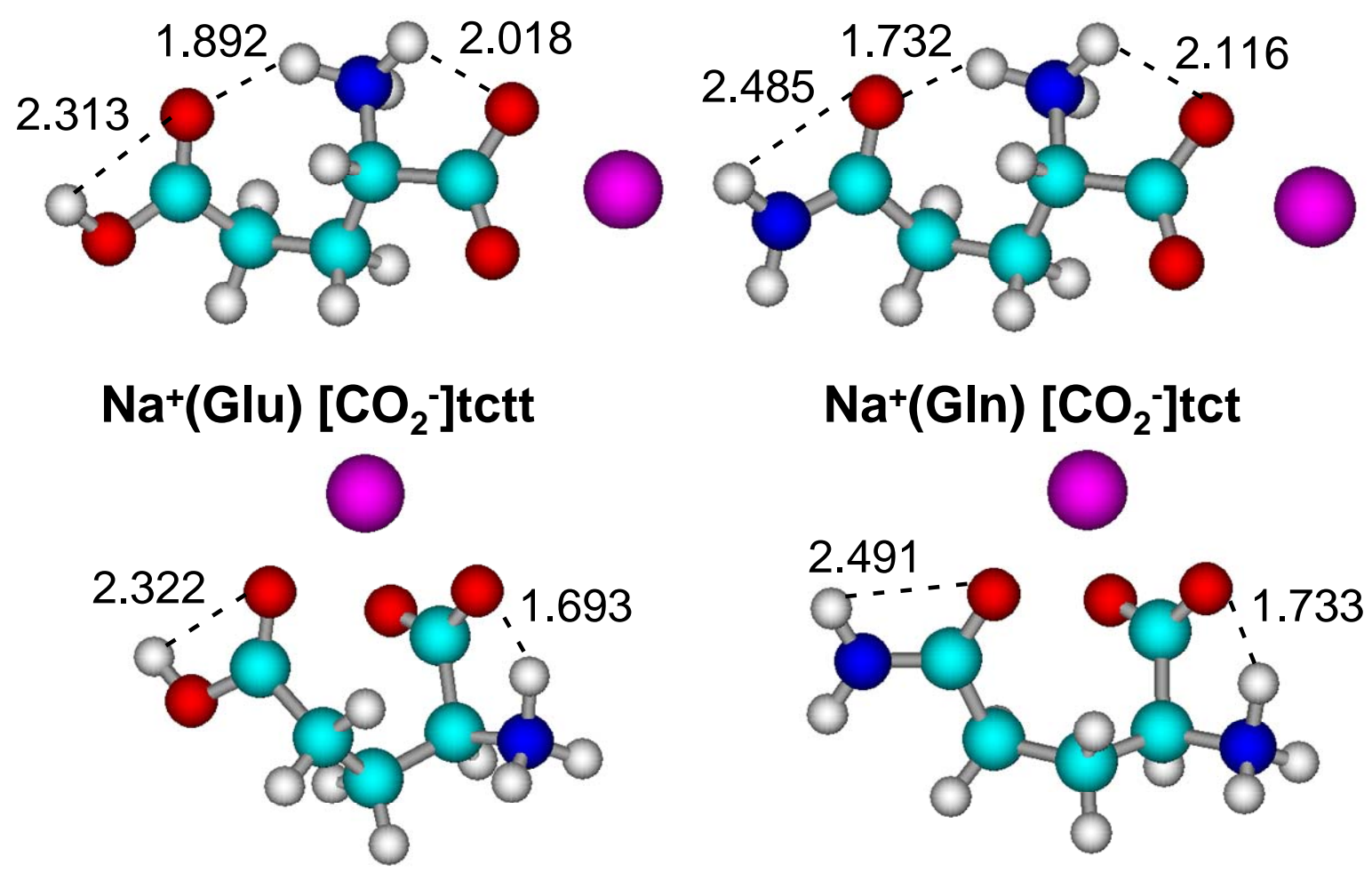

$\mathrm{Na}^{+}(\mathrm{Glu})\left[\mathrm{CO}_{2}^{-}, \mathrm{CO}\right] \mathrm{ggtt}$

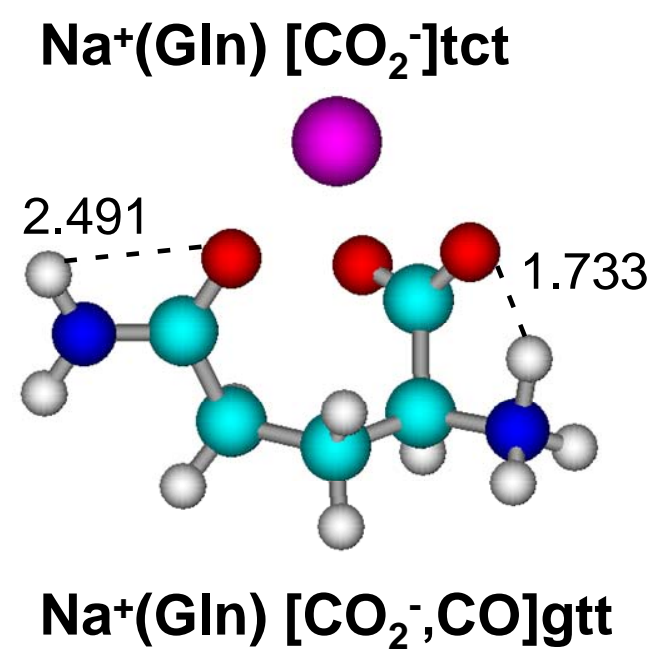

